\title{
Aprendizaje B-learning como enfoque mezclado no agitado con las teorías del aprendizaje
}

Fecha de recepción: 2021-09-01 • Fecha de aceptación: 2021-10-27 • Fecha de publicación: 2021-12-01

Florcita Janeth Arellano Espinoza ${ }^{1}$

Universidad Estatal de Bolívar, Ecuador

farellano@ueb.edu.ec

https://orcid.org/0000-0002-4325-9317

Danny Fernando Pérez Castillo² Universidad Estatal de Bolívar, Ecuador dperez@ueb.edu.ec https://orcid.org/0000-0003-4418-0050

Carlos Alfredo Gruezo González ${ }^{3}$ Universidad Estatal de Bolívar, Ecuador cgruezo@ueb.edu.ec https://orcid.org/0000-0002-5452-987X

Nancy Concepción Pérez Gaibor ${ }^{4}$ Universidad Estatal de Bolívar, Ecuador nperez@ueb.edu.ec https://orcid.org/0000-0003-0145-4912

\section{Resumen}

La evolución que se ha suscitado en los diferentes ámbitos (económicos, políticos, tecnológicos, sociales, educativos, religiosos, entre otros) de la humanidad, ha despertado especial interés en la implementación innovadora de sistemas educativos, sustentados en la evolución y practicidad 
que promueven las tecnologías de la información y comunicación (TIC), con miras a generar cambios drásticos que surgen de los diferentes elementos del acto didáctico. El objetivo del presente estudio es interpretar la importancia de los modelos Blended learning (B-learning) en los procesos de enseñanza educativo para la construcción del conocimiento, mediante el análisis de las teorías del aprendizaje. Es una investigación centrada dentro del paradigma postpositivistainterpretativo, enfoque cualitativo (interpretativismo-sociosimbolista) de tipo documental. Los resultados indican que, debido a la versatilidad que ofrece el diseño B-learning, en cuanto a su nivel integrador para estructurar sesiones presenciales y virtuales, en concordancias con las TIC y las teorías del aprendizaje; generan procesos innovadores y creativos que realzan la importancia de la disciplina, comunicación y aprendizaje; entre sus actores principales (profesores y estudiantes). Las conclusiones denotan el resurgimiento de fuentes de horizontes, enmarcadas bajo actividades de enseñanza, donde las instituciones ameritan enfocar sus estrategias en pro de fortalecer los sistemas educativos, volviéndose resilientes y capaces de ofrecer respuesta a la sociedad a pesar de las adversidades y aprovechando la tecnología.

Palabras clave: B-learning, innovación educativa, educación online, estrategias comunicativas, mixtura educativa, TIC

\section{Abstract}

The evolution that has occurred in the different areas (economic, political, technological, social, educational, religious, among others) of humanity, has aroused special interest in the innovative implementation of educational systems, based on the evolution and practicality promoted by information and communication technologies (ICT), with a view to generating drastic changes that arise from the different elements of the didactic act. The objective of this study is to interpret the importance of Blended Learning (B-learning) models in educational teaching processes for the construction of knowledge, through the analysis of learning theories. It is a research centered within the post-positivist-interpretative paradigm, qualitative approach (interpretivism-socio-symbolism) of documentary type. The results indicate that, due to the versatility offered by the B-learning design, in terms of its integrative level to structure face-to-face and virtual sessions, in accordance with ICT and learning theories; they generate innovative and creative processes that enhance the importance of discipline, communication and learning; among its main actors (teachers and students). The conclusions denote the resurgence of sources of horizons, framed under teaching activities, where institutions deserve to focus their strategies in favor of strengthening educational systems, becoming resilient and capable of offering a response to society despite adversities and taking advantage of technology.

Keywords: B-learning, educational innovation, online education, communicative strategies, educational mix, ICT 


\section{Introducción}

La educación, vista desde el marco de la filosofía, es fundada hacia la evolución de procesos formativos, donde la incomprensión de algunos que ejercen la profesión de docente, se ha visto descontextualizada de sus verdaderos ideales, desvirtuando el verdadero sentido filosófico del ámbito educativo; que tenía como norte para la generación de significados en el adiestramiento de formadores, por lo cual, la educación, desde la filosofía, aborda cuestiones filosóficas relativas a la naturaleza, los objetivos y los problemas de la educación (Martínez et al., 2021). Por otro lado, la formación académica es comprendida como una corriente direccionada a procesos que tienen relación con el conocimiento, aprendizaje y necesidades; según la contextualización y los entornos divergentes en el ramo educativo.

Como parte de esta formación, la sociedad de la información ha contribuido contundentemente en la producción de novedosas estrategias y metodologías, con la intención de ofrecer lo más práctico posible el acceso al conocimiento, promoviendo espacios dinámicos para la interacción e intercambio de ideas. En esta misma perspectiva, Monge y Contractor (2003) plantean que los diseños de redes se presentan como una alternativa viable para el acceso a la información, abriendo brechas positivas hacia la construcción del conocimiento; sobre todo en escenarios relacionados con la educación; permitiendo a los principales participantes (profesores y alumnado) interactuar y dinamizar los encuentros de enseñanza-aprendizaje mediante tiempos iguales o diferentes.

Asimismo, las universidades requieren masificar la información para permitir el acceso al conocimiento, considerando para ello el avance de la ciencia y los cambios generados en la sociedad. Es de esta forma como las nuevas tecnologías de la información y comunicación (TIC) emergen, debido a su capacidad para crear inéditas formas comunicacionales, empleando la microelectrónica, informática y telecomunicaciones; mediante herramientas tecnológicas y desarrollo cognitivo, con el fin de conceder acceso, emisión y procesamiento de la información (Díaz et al., 2021).

Cabe resaltar que en ciertas situaciones dentro de un proceso de enseñanza-aprendizaje, las TIC juegan un papel importante, dado al apoyo que surte a sistemas dinámicos mediante actividades didácticas que integran lo visual, novedoso e interactivo (García et al., 2018). Es considerada herramienta valiosa, sobre todo en esta época impregnada de información y conocimiento, por lo tanto, el sistema educativo se ve en la necesidad de incorporarlas, con el fin de facilitar a la sociedad mecanismos que coadyuven y aceleren el procesamiento de la indagación; sin embargo, no están articuladas en el proceso educativo.

Para complementar estos procesos interactivos, y acompañar el dinamismo de las TIC; se encuentran los sistemas Blended learning (B-learning) que son programas basados en la intersección comunicacional, aprovechando las ventajas y bondades de recursos provenientes del aprendizaje virtual, así como de la interacción y las sinergias generadas en los grupos en las sesiones presenciales y semipresenciales (Salinas et al., 2018). Representan una mezcla o modelo mixto, sin distinción pedagógica entre las clases presenciales y virtuales. 
En este sentido, la presente investigación pretende interpretar la importancia de los modelos $B$-learning dentro de los procesos de enseñanza y aprendizaje en la educación, es relevante para la construcción del conocimiento, presentando un análisis que se integre con las teorías del aprendizaje. Para lograrlo fue necesario revisar diferentes fuentes y trabajos científicos, que permitieron fundamentar las argumentaciones a partir del razonamiento y fusiones de horizontes epistémicos, con la finalidad de comprender e interpretar el funcionamiento y características de estos programas, en concordancia con las TIC, sobre todo desde la visión de los docentes y estudiantes; en búsqueda de fortalecer la enseñanza y el aprendizaje.

\section{El B-learning visto desde la investigación educativa}

Desde el momento que comenzó a emplearse, este término ha tenido diferentes denominaciones, tales como: aprendizaje híbrido, semipresencial, mixto y mezclado (Picciano, 2014; Morán, 2012). Así como existían varias contextualizaciones, al mismo tiempo se conocían diversas visiones como, por ejemplo, las destacadas por Tayebinik \& Puteh (2012), donde expresaban la combinación relacionada con el aprendizaje presencial-online y la mixtura estratégica de enseñanza. Lo cierto de todo esto es que generalmente se conjugaban con tendencias sólidas, híbridas, mixtas y combinadas; para contrarrestar las limitaciones educativas a distancia, promulgando la comunicación y la innovación de herramientas didácticas, con la intencionalidad de mejorar los sistemas educacionales.

El término B-learning, según González (2015), es un espacio virtual donde los profesores y alumnado se relacionan para llevar a cabo el proceso educativo, desde una plataforma sustentada en el Internet, creando la modalidad de enseñanza no presencial, virtual o en línea, es decir, es una zona o área donde se mezclan las perspectivas educativas presenciales y E-learning (educación online); constituyendo el aprendizaje conducido de tipo mixto, mejor conocida como B-learning.

Esto significa la convergencia entre dos entornos de aprendizaje. Por un lado, el presencial; que representa la continuidad y tradición de los sistemas educativos y, por el otro; el ámbito virtual, que expande y revoluciona las posibilidades de comunicación y de generar interacción en entornos de la enseñanza y aprendizaje. Asimismo, este último contribuye a generar procesos de comunicación; a pesar de las distancias y circunstancias.

En resumen, los programas basados en el ambiente $B$-learning tienen un enfoque de aprendizaje que combina la formación presencial impartida por un formador y las actividades de aprendizaje en línea. En los procesos educativos, tanto en la modalidad presencial, como en la modalidad virtual, contribuyen al fortalecimiento de las relaciones interpersonales entre docentes y estudiantes, Por tanto, la interacción adquiere importancia para el logro de los objetivos didácticos. Lo relevante de la herramienta tecnológica desde la óptica generada por el entorno, la provee de opciones multimétodos para facilitar la comunicación y diálogo entre los actores. Por el lado de los docentes, plantean dinámicas que invitan a la participación activa en las bondades que los softwares ofrecen al momento de adherirse y, finalmente; desde la postura de los estudiantes, hacen uso de las herramientas para expresar sus ideas y desarrollar el aprendizaje (Bonilla et al., 2018). 


\subsection{Teorías del aprendizaje que sustentan la herramienta B-learning}

Estas pretenden describir los procesos mediante los cuales los seres humanos aprenden (Morinigo \& Fenner, 2021). Estas ayudan, refuerzan los autores; a comprender, predecir y controlar el accionar humano; elaborando a su vez estrategias de aprendizaje y tratando de explicar cómo los sujetos acceden al conocimiento. Su objeto de estudio se centra en la adquisición de destrezas y habilidades en el razonamiento y en la adquisición de conceptos.

Con el fin de ofrecer una base empírica sólida, los estudios actuales sobre el aprendizaje se centran más en elaborar teorías y en lograr descripciones detalladas con la conducta en situaciones concretas.

\subsubsection{Teoría cognitiva}

En sus inicios (década de los años 50), la teoría del aprendizaje comenzó a tomar distancia sobre el uso de los modelos conductistas, direccionándose hacia un enfoque proveniente de las ciencias cognitivas. De este modo, los educadores y psicólogos dejaron saber su interés e inclinación por conocer y emplear a fondo procesos cognitivos complejos, tales como: la solución de problemas, lenguaje, procesamiento de la información y formación de conceptos (Nobre et al., 2020).

Los autores mencionan que el aprendizaje bajo este esquema del cognitivismo; se encuentra vinculado a la manera de cómo el estudiante lo desea adquirir. Por medio de la teoría cognitiva se logra describir la adquisición del conocimiento derivada de un proceso mental que conlleva una codificación interna, que es diseñada y planeada por el estudiante. Bajo esta teoría, el alumno es entendido como un sujeto activo que procesa la información y es capaz de construir significados. Se enfoca en un nivel del procesamiento de la información y en la programación de ordenadores para realizar tareas cognitivas.

Cada vez que se le presente un conflicto cognitivo, el estudiante busca un nuevo equilibrio, incorporando la interacción social y la toma de conciencia en la solución de problemas. De esta forma, el aprendizaje se transforma en el fruto del desarrollo de estructuras tales como la formación, elaboración, organización y funcionamiento operacional, que no deben ser transpuesta directamente a la pedagogía.

\subsubsection{Teoría por descubrimiento}

Tiene sus raíces en la manera cómo se canaliza el pensamiento creativo y productivo. La manera idónea para desarrollarlo en el alumnado es ofreciendo alternativas y orientaciones hacia la curiosidad, de forma que estimule la investigación, indagación y planteen soluciones ante las adversidades. Molina (2020) sostiene que lo más importante es que el estudiante entienda el proceso por el cual ha llegado al conocimiento, y determine e interprete, la relación causa-efecto generado durante la actividad.

En conjunto, si el docente programa actividades donde el alumnado tenga que emplear el razonamiento y la imaginación; entonces está fomentando el desarrollo de su inteligencia y 
creatividad. De esta manera, el estudiante podría llegar a niveles donde se impulse a aprender y participar de forma dinámica, por la sencilla razón de querer obtener más aprendizaje. Hacia estas tendencias es que los sistemas bajo un enfoque B-learning deben plantearse, para impulsar procesos novedosos y que de manera espontánea surjan alternativas y compartan experiencias mixtas (docente-aprendiz); para el resurgir de soluciones a problemas planteados en la comunidad.

\subsubsection{Teoría constructivista}

El constructivismo es una teoría que realiza analogías entre el aprendizaje y el diseño de significados, tomando en consideración el empirismo y las situaciones que cada sujeto vive. Boggino (2004) plantea que, aun cuando el constructivismo se le considera como una rama del cognitivismo, ambos enfoques comprenden el aprendizaje como una acción mental, donde los psicólogos creen que la mente es una herramienta de referencia para el mundo real, por lo tanto, cada ser filtra en su mente lo que le llega del mundo exterior y, de esa manera, construye su propia realidad.

En este sentido, es significativo expresar que aun cuando en las instituciones educativas se están haciendo esfuerzos por aplicar este enfoque en el desarrollo de la enseñanza, no se entiende porque existe una tendencia de parálisis paradigmática que tienen algunos docentes y alumnos, para ser constructores del aprendizaje. Hay que separarse de ese sistema tradicional, en donde el agente activo es el docente y el alumno sigue en una actitud pasiva que simplemente asume al modelaje del profesor.

\subsection{Enfoque interactivo}

La teoría de Vygotsky (1988) presenta al aprendizaje con un alto contenido social, colectivo y comunitario, progresista y evolutivo; en concordancia con un concepto de inteligencia íntimamente ligado a la interacción y comunicación. De allí que a esta teoría se le denomina formación social de la mente, cuya característica principal se fundamenta en el desarrollo cognitivo, no sólo como producto biológico (genético), sino esencialmente social.

En este enfoque el profesor actúa como un mediador del aprendizaje, planificando actividades y empleando los recursos necesarios para poder llevar a cabo el proceso de enseñanza. Al contrario, los estudiantes, factor primario en esta teoría, toman una actitud activa para cristalizar el aprendizaje emprendido. En esta perspectiva, el pedagogo no tiene un papel protagónico, es el estudiante quien pasa al frente, donde el proceso de aprendizaje se realiza de forma constructiva, permitiendo hacer distinciones en cuanto a los tipos de conocimiento y las relaciones que se establecen.

\section{Aprendizaje combinado o B-learning como teoría}

El proceso de aprendizaje en las denominadas aulas o entornos virtuales se realiza sin restricción de tiempo, horario y lugar. El aprendizaje B-learning es definido por Bolívar (2007) como un modo de enseñanza, en la cual el docente combina el rol tradicional de la modalidad presencial con el rol de mediador en la modalidad a distancia, con uso intensivo de las tecnologías. En otras palabras, 
la modalidad semipresencial o aprendizaje combinado, es aquella donde existen encuentros tanto físicos o presenciales, como virtuales, y donde se usan como apoyo las TIC o recursos electrónicos, tales como foros, chat, correo electrónico, entre otros.

Recapitulando lo más importantes de estas teorías planteadas, un ambiente de aprendizaje es aquel lugar donde los estudiantes intercambian opiniones, puntos de vistas, experiencias bajo condiciones sociales, físicas, humanas y culturales; que se combinan para crear experiencias de formación significativa. Estas experiencias son el producto de actividades y estrategias diseñadas, acompañadas y desarrolladas por un docente, teniendo como base algunas teorías planteadas. Estas nuevas tendencias de la enseñanza deben estimular el aprendizaje autónomo y su correspondiente responsabilidad por cada uno de los estudiantes, siendo uno de los objetivos que se persigue en el andamiaje del aprendizaje B-learning.

\section{Metodología}

El estudio es de tipo documental con una estructura de revisión sistemática y con un paradigma postpositivista-interpretativo. Se logró identificar la revisión epistémica de los ejes temáticos, verificando la evidencia y credibilidad mediante el empleo del método hermenéutico, que pretende conocer las realidades con las que convive, ya sea empleando textos orales o escritos, analizando sus conductas, o estudiando los gestos de cualquier naturaleza que expresan vida.

El desarrollo de esta investigación se centra en el paradigma postpositivista-interpretativo, el cual Martínez (2015) señala que tiene entre sus características más resaltantes, cuestionar la existencia de una realidad externa y valiosa para ser analizada, dinámica, múltiple y holística. Asimismo, el enfoque epistémico es cualitativo (interpretativismo-sociosimbolista) sustentado en Guzmán (2017), quién señala que parte de la conjunción entre el origen del conocimiento y la relación con el objeto que se conoce. Es decir, se entrelazan el racionalismo (raíz del entendimiento) con el idealismo (comportamiento de los objetos, según la postura de quien los mire) con la intención de generar una relación entre el individuo y la realidad, emergiendo tendencias subjetivistas entre el ser y la fuente del conocimiento.

Como estrategia de interpretación, se compararon con la herramienta $B$-learning teorías del aprendizaje y dos herramientas tecnológicas de enseñanza como lo son el Blackboard learn y Canvas, que sirvieron de parámetros para conocer el nivel de incidencia, factibilidad, eficiencia y efectividad de su aplicación en el mundo de la educación virtual.

\section{Resultados}

Interpretando un concepto, partiendo de los enfoques investigados, se puede decir que el aprendizaje $B$-learning representa una herramienta que tiene la propiedad para integrar 0 compartir, en concordancia con las TIC, diferentes estrategias de enseñanzas cuya combinación tiene ciertas prevalencias con lo presencial y lo virtual (ver Figura 1). 
Figura 1.

Proceso de integración B-learning con las teorías del aprendizaje

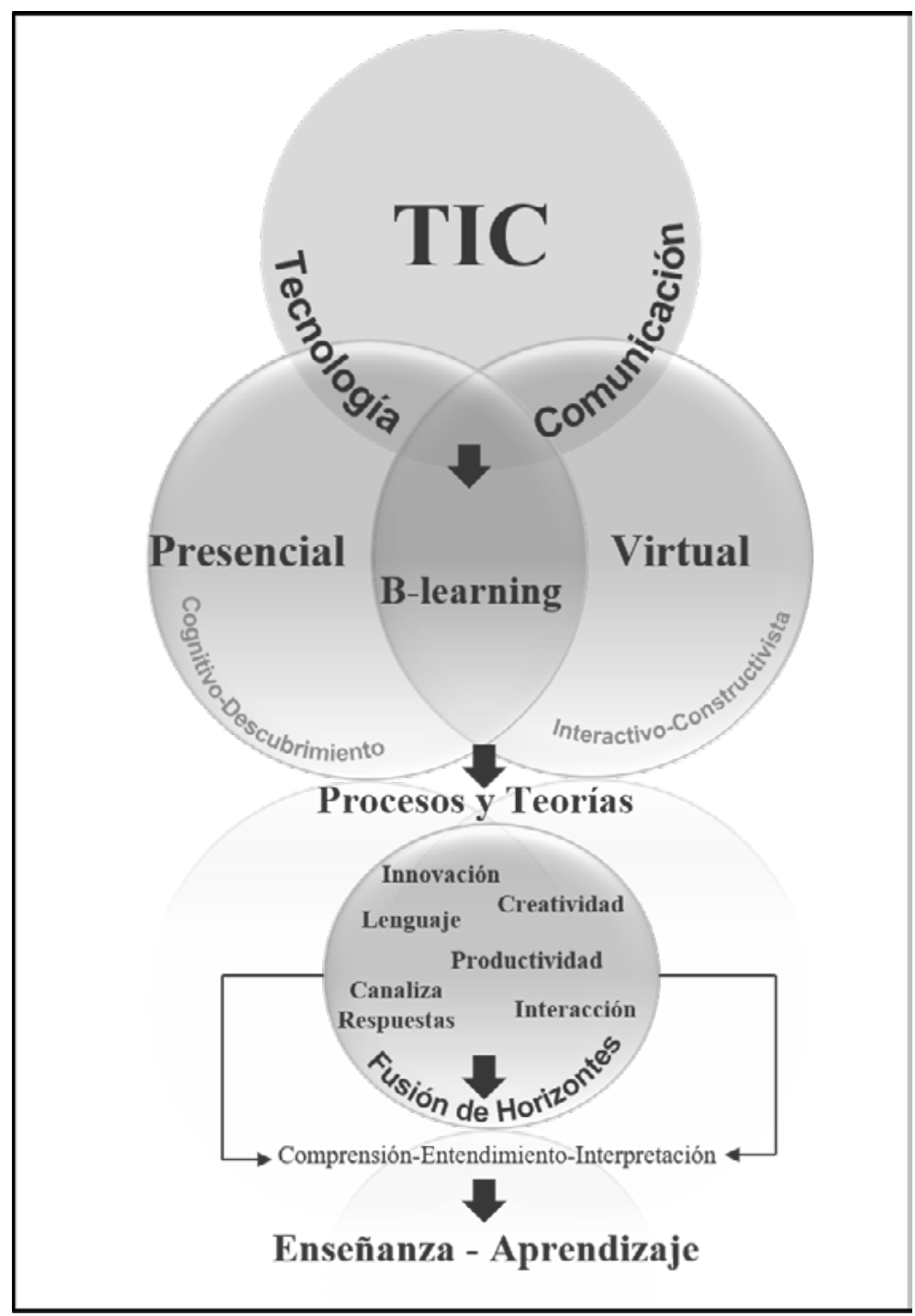

Esto demuestra la importancia que pueden ejercer las diferentes teorías del aprendizaje, la cual deben tener presente todos aquellos programas $B$-learning enfocados en el sistema educativo. Es necesario aprovechar todas las características que ofrece la tecnología, e integrarla con las interacciones que han venido funcionando con los procesos de enseñanza, para reducir al máximo los errores que pudieran surgir en el aprendizaje, como objetivo directo que se persigue.

De esta manera, el constructivismo busca simplificar la conducta humana, observando al ser como una automatización en lugar de una criatura con propósito y voluntad. Por lo tanto, coadyuva en el sistema educativo al impulso de la experimentación científica, y al uso de procedimientos estadísticos. Su importancia radica en la interacción con el ramo de la psicología, como herramienta para formular soluciones verdaderas, relacionadas con la conducta humana. La forma en que el $B$-learning se puede apoyar con la teoría constructivismo permite analizar 
el comportamiento de los estudiantes hacia la aceptación del medio tecnológico, debido a las facilidades o bondades que deben existir para incitar al uso o empleo de la plataforma de enseñanza.

El sistema de aprendizaje, visto desde esta fusión con las TIC, puede ser de gran utilidad constructivista, tanto para los profesores, como para las instituciones educativas. La influencia viene arraigada a lo predominante de esta teoría hacia la educación, debido a la manera de cómo se ven los estudiantes como individuos vacíos que adquieren conductas desalineadas con el proceso de aprendizaje, pudiendo ser reemplazadas o eliminadas; admitiendo la gran influencia de la educación tradicional y la nueva forma de visionar el novedoso sistema de aprendizaje y evaluación.

Por el lado de la teoría cognitiva se han dedicado de manera especial al estudio de los procesos de la memoria (y de los procesos relacionados con ella: atención, percepción, lenguaje, razonamiento y aprendizaje). Para ello, parten de la creencia de que es la mente la que dirige a la persona, y no los estímulos externos, donde el sujeto es considerado un ser capaz de dar sentido y significatividad a lo que aprende (un procesador de información). Ya acá se puede observar la influencia o significancia que esto genera desde la óptica de los B-learning, el cual un sistema debe ser capaz de recibir la información, procesarla, elaborar planes de actuación, tomar decisiones, ejecutarla y evaluarla.

La teoría se conjuga con las TIC, para producir un sistema de reajuste comunicativo, el cual puede ser llamado perspectiva del procesamiento de la información, donde desde una postura cognitivista, las relaciones que se establecen entre lo conocido y lo nuevo, son la base del aprendizaje. En este sentido, la esencia de la adquisición del conocimiento consiste en aprender a establecer relaciones generales, que permita ir engranando unos conocimientos con otros, y por ello, el aprendizaje requiere construirse con sabiduría, conectando las informaciones nuevas, con la que teníamos anteriormente, hasta producirse procesos recursivos si se analiza desde una postura compleja.

Al analizarse el $B$-learning con la teoría interactiva, aprovechando la versatilidad que se ajusta a procesos presenciales y virtuales, se desprenden una serie de características entre las que se resaltan que a la modalidad semipresencial se le pueden aplicar diversas técnicas y metodologías de enseñanza, permitiendo interactuar e intercambiar bien sea con grupos o de forma individual, contribuyendo al desarrollo de habilidades de enseñanza educativa entre todos los estudiantes que usan esa modalidad. Asimismo, permite estructurar diseños flexibles, donde cada alumno tenga la iniciativa de organizar el tiempo necesario y justo, para desarrollar las actividades que son impartidas por los profesores. La interacción debe ser vista por los sistemas B-learning, como una herramienta que en lugar de convertirse en elemento distanciador, más bien sea integrador y proveedor de impulso hacia la enseñanza.

La teoría interactiva, permite entre otras cosas, que el estudiante cuente con el apoyo del profesor, así como también con la colaboración de otros participantes. Por lo tanto, al momento de emplearse con las TIC, se fusionan creando un recurso complementario de respaldo y sustento de contenidos para apoyar las clases virtuales, convirtiéndose en un recurso versátil para el 
proceso de aprendizaje, alcanzándose un alto número de objetivos educativos planteados por los facilitadores. En este punto es preciso aclarar que, una de las bondades del B-learning consiste en la facilidad intrínseca que posee, para crear materiales educativos digitales en diferentes formatos.

Cómo se puede notar, la teoría interactiva se centra básicamente en el estudiante, debido a que representa el ente principal y responsable dentro del proceso de aprendizaje mezclado no agitado. Este tipo de aprendizaje mixto logra estimular el desarrollo del pensamiento crítico, pues los estudiantes, al exponer sus ideas a través de esta teoría en concordancia con las TIC, adquieren un sentido colaborativo y autónomo. Es bueno acotar que el docente actúa como un mediador en el proceso de aprendizaje.

Finalmente, los resultados hermenéuticos arrojados en la investigación referente a la teoría del descubrimiento; se enfila en el pensamiento creativo y productivo. Las alternativas relacionadas con los softwares existentes en el mercado, la cual tratan de enmarcarse y cubrir todas las necesidades que exigen y necesitan las instituciones bajo este esquema B-learning y las TIC, vienen a representar una de las herramientas digitales que formarían parte de los novedosos sistemas de aprendizaje y enseñanza educativa.

A continuación, se detallan y describen las herramientas Blackboard learn y Canvas; seleccionadas para este trabajo, donde se abordan las características distintivas y su utilidad dentro del ámbito de la enseñanza académica.

\subsection{Blackboard learn}

Es conocido como uno de los softwares más relevantes dentro de la gestión del aprendizaje en línea (LMS, por sus siglas en inglés Learning Management System). Resulta ser bien práctico en cuanto al nivel de interacción, permitiendo compartir la información y comunicación entre tutores y estudiantes, en otras palabras, resulta ideal para los aprendizajes grupales (Suliman y Muhammad, 2021). Una de las principales ventajas del Blackboard learn es que ofrece tecnología de punta a los usuarios, confirmando sus preferencias en el entorno académico, por ser un sistema efectivo y eficiente para el manejo del aprendizaje LMS.

En palabras de Villalón et al. (2019), la importancia de esta herramienta viene dada en lo compatible que resulta la plataforma respecto a los tres sistemas operativos más comunes (Microsoft Windows, Mac OS X y Linux). Aunado a esto, tiene la posibilidad de integrarse con otros entornos de aprendizaje como, por ejemplo, Moodle. Es importante acotar que permite la comunicación síncrona por medio de videoconferencias, mensajería instantánea y transmisión de voz; logrando fusionar el compartimiento de diferentes archivos y la admisión de contenido multimedia.

Entre las características distintivas del software se destacan las siguientes: cuenta con siete plataformas formativas (Learn, Transact, Engage, Connect, Mobile, entre otros), ofrecidas por Bundled software. También, la plataforma es utilizada en múltiples países (desde el año 2014 se extiende aproximadamente a 17000 escuelas y organizaciones en 100 países). El 75\% de las universidades en Estados Unidos y más de la mitad de sus escuelas, usan este producto. 
Tiene consigo diferentes aplicaciones propias para dictar clases en línea, que son de mucha utilidad para diseñar los cursos online. Entre las múltiples alternativas que tiene disponible se señalan las siguientes: tiene una pizarra, realiza videoconferencias, sistema de exámenes en línea, los alumnos puedan elaborar y presentar sus trabajos online, chat privado y grupal, crea foros de discusión y viene acompañado con un soporte permanente ante cualquier problema que pueda presentarse, desde el punto de vista técnico.

\subsection{Canvas}

Es un software evaluativo tipo LMS, que sirve para rastrear y proporcionar información sobre la enseñanza de los estudiantes en los cursos y en un programa académico. Tiene la capacidad de recopilar puntajes de logros para los resultados del aprendizaje, basados en criterios evaluables de las tareas de evaluación integradas en los cursos y otras oportunidades, a través de las cuales los estudiantes demuestran competencias (Burrack \& Thompson, 2021).

Esta plataforma se orienta hacia lo estético por la facilidad de uso, no obstante, entre las principales características se denotan: es enfocada en perfiles de usuarios para poder configurar los roles de aprendizaje, contiene temas gráficos para integrar y personalizar, calendarios, E-learning sincrónicos y asincrónicos, Dashboard (indicadores de progreso durante el sistema de aprendizaje), es de orientación social, individual y colectivo, compatibilidad con contenido SCORM (por sus siglas en inglés de Shareable Content Object Reference Model), integra herramientas de interoperabilidad de equipos de aprendizaje (LTI), incluye historial académico, creación y gestión de correos internos y es compatible con aplicación Móvil Android y IOS.

En definitiva, la plataforma LMS Canvas tiene como fundamento facilitar el proceso de aprendizaje, tanto a estudiantes, como a profesores. Para ello es reconocido como sencillo, práctico y bastante amigable, ya que mantiene un ambiente manejable y accesible para los usuarios.

Lograr que las herramientas basadas en ambiente $B$-learning, puedan sustentarse tomando en consideración algunas de las teorías del aprendizaje expuestas (pueden adherirse otras), permiten que el sistema se fortalezca en pro de resaltar las nuevas tendencias enmarcadas en los procesos TIC, con la intención de apoyar la enseñanza y el aprendizaje; ya no solo desde una perspectiva presencial, sino combinándolo y obteniendo mejores resultado, adaptándose a las nuevas eras que ofrece el ciberespacio.

\section{Conclusiones}

La innovación consiste en atreverse a diseñar o estructurar algo distinto en búsqueda de diferentes resultados o expectativas tradicionales. Viene acompañado de procesos creativos que buscan adaptar nuevos enfoques basados en las necesidades o circunstancias que se presentan, con la intencionalidad de presentar alternativas que permitan salir adelante $u$ ofrecer respuestas satisfactorias ante cualquier evento o complejidad. Son procesos que pudieran llamarse resilientes, ya que su fisonomía otorga resultados y aprendizaje derivados de las experiencias vividas. 
Es así como funciona el aprendizaje bajo plataforma tecnológica $B$-learning, como enfoque mezclado no agitado con las teorías del aprendizaje, donde se infiere que la importancia, relevancia y participación de estos modelos en concordancia con las TIC, determinando el grado de preponderancia necesario para el fortalecimiento de actividades de enseñanza educativa, que posibilita la construcción del conocimiento mediante el análisis de las teorías expuestas que sirven de apoyo para el proceso comunicativo entre los principales actores (profesores y los alumnos).

La fusión de horizontes intervinientes en el proceso y provenientes de las teorías, dan acceso a la activación de tareas con expectativas o intenciones básicas, donde deben ser fortalecidas por medio del entendimiento de los objetivos que se deben establecer, para diseñar programas bajo la plataforma B-learning, que fortalezcan al aprendizaje y enseñanza en el ámbito educativo bajo el sustento de la teoría constructivista. Una vez que se logra esa fusión, es importante internalizarla, interpretarla y adaptarla; a las necesidades y realidades que envuelven al sistema educacional, con miras a la actual era moderna basada en la tecnología y en las nuevas estrategias educacionales. 


\section{Referencias}

Boggino, N. (2004). El constructivismo entra al Aula. Editorial Homo Sapiens.

Bonilla Jurado, D., Oña Sinchiguano, B., \& López Núñez, H. (2018). Medición de innovación tecnológica como eje central del crecimiento empresarial familiar del sector carrocero de la Provincia de Tungurahua. Revista Lasallista de Investigación, 15(2), 271-285. https://doi.org/10.22507/rli.v15n2a21

Bolívar, C. (2007). El Blended Learning: evaluación de una experiencia de aprendizaje en el nivel de posgrado. Teoría de la Educación. Educación y Cultura en la Sociedad de la Información, 8(3), 188-199. https://www. redalyc.org/pdf/2010/201017307010.pdf

Burrack, F., \& Thompson, D. (2021). Canvas (LMS) as a means for effective student learning assessment across an institution of higher education. Journal of Assessment in Higher Education, 2(1), 1-19. https:// doi.org/10.32473/jahe.v2i1.125129

Díaz, J., Ruiz, A., y Egüez, C. (2021). Impacto de las TIC: desafíos y oportunidades de la Educación Superior frente al COVID-19. Revista Científica UISRAEL, 8(2), 81-92. https://doi.org/10.35290/rcui.v8n2.2021.448

García, M., Reyes, J., y Godínez, G. (2018). Las TIC en la educación superior, innovaciones y retos. RICSH Revista Iberoamericana de las Ciencias Sociales y Humanísticas, 6(12. https://doi.org/10.23913/ricsh. v6i12.135

González, M. (2015). El B-learning como modalidad educativa para construir conocimiento. Opción, 31(2), 501531. https://www.redalyc.org/pdf/310/31045568029.pdf

Guzmán, R. (2017) Paradigmas e investigación performativa. Un territorio epistémico-discursivo en educación. Masfe ediciones.

Martínez, H., Piña, D., y Martínez, J. (2021). Filosofía universitaria: una corriente para la construcción de significados en la formación de formadores. Revista Científica UISRAEL, 8(2), 81-92. https://doi.org/10.35290/ rcui.v8n2.2021.374

Martínez, M. (2015). Ciencia y arte en la metodología cualitativa. Editorial Trillas.

Molina, M. (2020). El aprendizaje por descubrimiento. Un cambio metodológico para aprender Didáctica de la Historia. Innovación Educativa, (30), 169-183. https://doi.org/10.15304/ie.30.6861

Monge, P., Contractor, N. (2003). Theories of communication networks. Oxford University Press.

Morán, L. (2012). Blended-learning. Desafío y oportunidad para la educación actual. Edutec, Revista Electrónica 
de Tecnología Educativa, (39), 1-19. http://dx.doi.org/10.21556/edutec.2012.39

Morinigo, C., y Fenner, I. (2021). Teorías del aprendizaje. Minerva Magazine of Science, 9(2), 1-36. https://www. minerva.edu.py/articulo/374/

Nobre, F., Juliane Amorim, J., y Leite, S. (2020). El desarrollo del aprendizaje a la luz de la teoría cognitiva. Revista de Ciências Humanas, Frederico Westphalen-RS, 21(2), 3-10. http://revistas.fw.uri.br/index.php/ revistadech/article/view/3560/pdf

Picciano, A. (2014). Introduction to Blended Learning: research perspectives. Routledge.

Salinas, J., De Benito, B., y Pérez, A. (2018). Blended Learning, más allá de la clase presencial. Ried Revista Iberoamericana de Educación a Distancia, 21(1), 195-213. http://dx.doi.org/10.5944/ried.21.1.18859

Suliman, A., y Muhammad, R. (2021). The use of M-Learning: A perspective of learners' perceptions on M-Blackboard Learn. International Journal of Interactive Mobile Technologies, 15(2), 4-17. https://doi.org/10.3991/ ijim.v15i02.17003

Tayebinik, M., y Puteh, M. (2012). Blended Learning or E-learning? International Magazine on Advances in Computer Science and Telecommunications, 3(1), 103-110. http://doi.org/10.1016/j.iheduc.2012.12.00

Villalón, R., Luna, M., y García-Barrera, A. (2019). Valoración y uso de la plataforma Blackboard en una universidad a distancia: estudio de caso sobre las prácticas declaradas de docentes del grado de Psicología. Digital Education Review, (35), 267-288. https://doi.org/10.1344/der.2019.35.267-288

Vygotsky, L. (1988). El desarrollo de los procesos psicológicos superiores. Editorial Grijalbo. 
Copyright (c) 2021 Florcita Janeth Arellano Espinoza, Danny Fernando Pérez Castillo, Carlos Alfredo Gruezo González y Nancy Concepción Pérez Gaibor

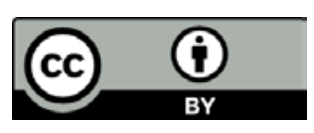

Este texto está protegido bajo una licencia internacional Creative Commons 4.0.

Usted es libre para Compartir-copiar y redistribuir el material en cualquier medio o formato

- y Adaptar el documento - remezclar, transformar y crear a partir del material-para cualquier propósito, incluso para fines comerciales, siempre que cumpla las condiciones de Atribución. Usted debe dar crédito a la obra original de manera adecuada, proporcionar un enlace a la licencia, e indicar si se han realizado cambios. Puede hacerlo en cualquier forma razonable, pero no de forma tal que sugiera que tiene el apoyo del licenciante o lo recibe por el uso que hace de la obra.

\section{$\underline{\text { Resumen de licencia - Texto completo de la licencia }}$}

